\title{
The Effect of the Change in Neck Position on Muscle Activity during Arm Flexion Exercise
}

\author{
Sung-Hak Cho, PT, PhD $\cdot$ Chi-Hwan Kim PT, $\mathrm{PhD}^{1 \dagger}$
}

Department of Physical Therapy, Kaya University, ${ }^{1}$ Department of Physical Therapy, Jaseng Hospital of Korean Medicine

Received: November 25, 2020 / Revised: November 28, 2020 / Accepted: December 03, 2020

(C) 2021 J Korean Soc Phys Med

\section{| Abstract |}

PURPOSE: This study was to investigate the effect of applying neck flexion during elbow flexion exercise on muscle activity of the biceps brachii, the anterior deltoid and the sternocleidomastoid (SCM).

METHODS: Twenty healthy adults participated in this study. The subjects measured \%MVIC (Maximal volumetric control) and collected the maximum electromyography (EMG) values in the biceps brachii, anterior deltoid, SCM. The first action was to sit in a sitting position with the eyes facing front and maintained the weight of each section by performing elbow flexion using a tension gauge. The second action was to sit in a sitting position with the head down and maintained the weight of each section by performing elbow flexion using a tension gauge. Paired T-test was used for comparison of the before-and-after neck flexion to analyze the muscle activity of the Biceps brachii of next flexion and

$\uparrow$ Corresponding Author : Chi-Hwan Kim ace1521@naver.com, https://orcid.org/0000-0001-7133-5658 This is an Open Access article distributed under the terms of the Creative Commons Attribution Non-Commercial License (http://creativecommons.org/licenses/by-nc/3.0) which permits unrestricted non-commercial use, distribution, and reproduction in any medium, provided the original work is properly cited. extension, the SCM, and the Anterior deltoid during elbow flexion exercise. The Repeated measured ANOVA was used for comparing $\% \mathrm{MVIC}$ in each muscle. The significance level was set at .05 .

RESULTS: Neck flexion during elbow flexion increased the muscle activity of the biceps brachii and anterior deltoid muscle in the maximal muscle strength $50 \sim 60 \%, 70 \sim 80 \%$ and decreased the muscle activity of the $\operatorname{SCM}(\mathrm{p}<.05)$.

CONCLUSION: The applic has been confirmed to reduce the mobilization of the SCM and increase the mobilization of the biceps brachii and anterior deltoid to increase the mobilization. Therefore, it seems effective to apply neck flexion when elbow flexion exercise.

Key Words: Elbow flexion exercise, Neck flexion, EMG, Biceps brachii

\section{Introduction}

Arms are one of the most used human body in daily life [1]. The lack of the function for the flexion and the extension of arms brings huge inconvenience to our daily life [2]. In particular, problems of the action of the elbow joint brings about restrictions in motion such as eating, dressing, washing, and writing etc [3]. To improve the 
muscular strength, various methods of exercise are applied and the fundamental principle of these exercises is the application of resistance [4]. Resistance training is the most effective exercise among a variety method of training [5]. Resistance training is effective in improving muscle strength, endurance, and preventing adult diseases [6], and the most commonly used to improve the physical strength of the general public and to train the physical strength of athletes by using sporting goods such as barbell, dumbbell, and machine [7]. The Biceps brachii is a muscle to connect the shoulder and forearm. The Biceps brachii is a muscle for flexion of arm and medial rotation. The SCM works as side bending and rotation of neck. The Anterior deltoid works as lifting arms to the side [8]. Barbell Curl is a representative exercise for arm flexion exercise. Barbell Curl is an exercise that repeating flexion and extension of forearm segment with elbow joint as an axle of rotational exercise with holding the barbell while standing upright. And also It is an exercise that affects the Biceps brachii and Brachialis and also overloads Brachioradial muscle, Pronator teres muscle, and flexor of fingers and wrist [9]. Selective strengthening is important to strengthen weakened muscles, and excessive resistance can lead to excessive contraction of other synergic muscles [10]. Also, the muscular activity of the synergic muscles will be affected depending on the exercise method and posture [11]. In order to improve the effect of the resistance exercise, resistance should be set at the fitness level of the athlete. Because not only the size of the power that can be exerted in the full range of exercise is different for each person, but also the power that can be exerted depending position and speed [6]. Many of physical director guide neck flexion exercise in order to create stronger strength during arm flexion exercise. Neck flexion often occurs due to excessive contraction of other synergic muscles by excessive resistance during elbow flexion exercise. However, data on how neck flexion affects during elbow flexion exercise is insufficient. This study was to search the effect of applying neck flexion during elbow flexion exercise on muscle activity of the Biceps brachii, the Anterior deltoid and the SCM.

\section{Methods}

\section{Subject}

Twenty selected men and women subjects performed this study and every participant were based on dominant arm for measurement. This study was approved by Bioethics committee of Kaya University (Kaya IRM-265).

Selection Criteria: Subjects were selected as a person who understand the explanation of the purpose and method of the study and agreed the participate this experience with healthy upper body with no problem on musculoskeletal system, and no pain within 3 months.

Exclusion Criteria: Subjects were excluded as a person who had experience a surgery in shoulder or upper limb and had received hospital treatment for shoulder pain within the last 3 months, and currently have pain in shoulder.

\section{Measurements}

\section{1) 4 Channel Wireless EMG}

To measure the muscle activity of the SCM and Upper limb flexors, a wireless EMG system (MA, Delsys Inc. USA) composed of 4 channels and a 4DMT-2EM.3.3 software analysis version of data analysis MG works were used for the experiment (Fig. 1). EMG electrodes were attached on the surface of the Biceps brachii, SCM, and the Anterior deltoid.

\section{2) Strength Analysis Device (Pul)}

This device consists of two straps and a strength analysis main body capable of connecting two strap. With using the strength analysis device, the section was set of maximal muscle strength $50 \sim 60 \%, 60 \sim 70 \%, 70 \sim 80 \%$. During the elbow flexion exercise, one of the strap was fixed by 


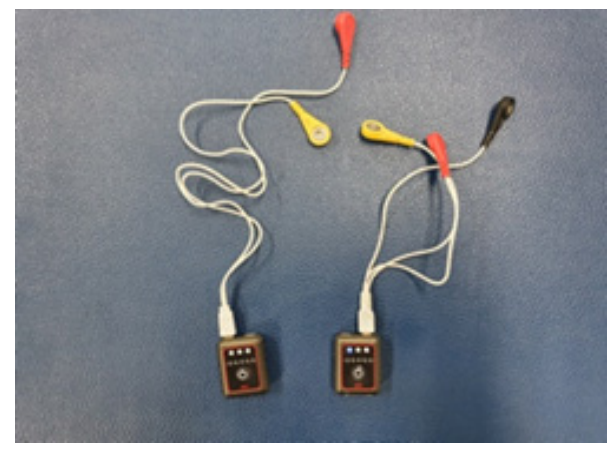

Fig. 1. EMG.

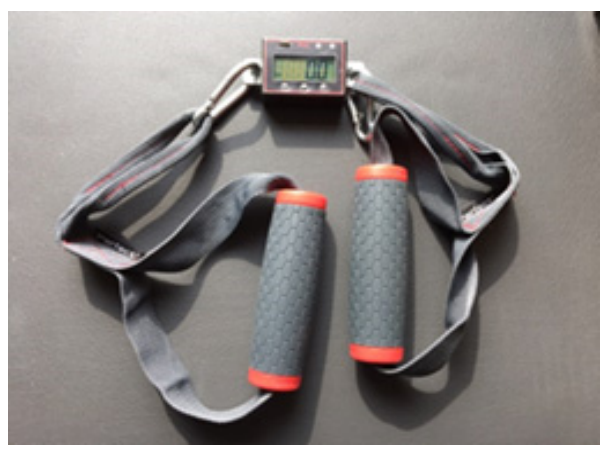

Fig. 2. Strength analysis device.

stepping on the foot, and the other was grabbed by hand for exercise (Fig. 2).

\section{Exercise Method}

Maximum EMG value of each muscle was obtained with performing \%MVIC (maximal voluntary isometric contraction) to each muscle. To collect the \%MVIC data, the following actions were performed. The first action was to sit in a sitting position with the eyes facing front and maintained the weight of each section by performing elbow flexion using a tension gauge (Fig. 3).

The second action was to sit in a sitting position with the head down and maintained the weight of each section by performing elbow flexion using a tension gauge. The neck flexion was not excessive (Fig. 4).

To maintain an accurate posture during performing the action, the subject conducted this experiment after twice

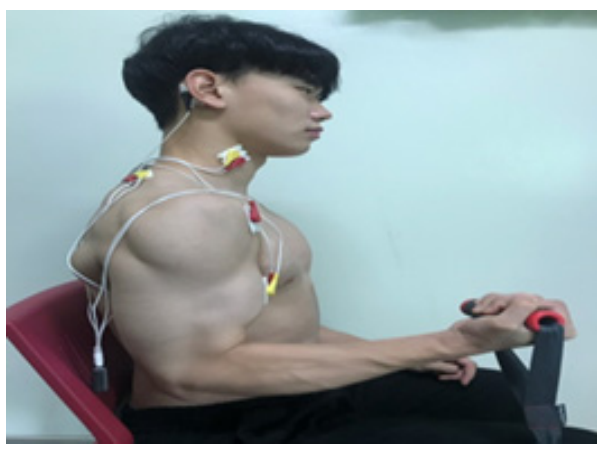

Fig. 3. Neck neutral-biceps curl exercise.

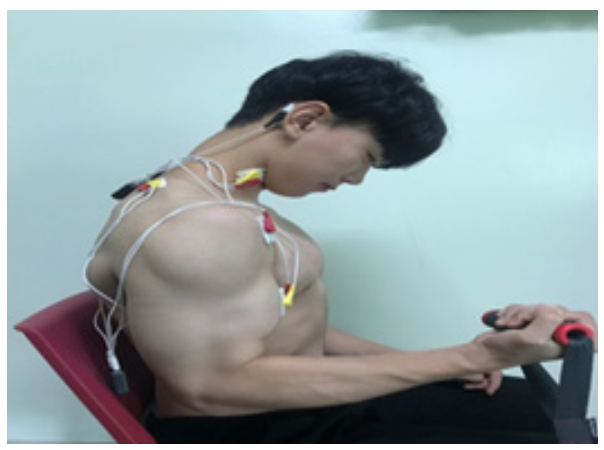

Fig. 4. Neck flexion-biceps curl exercise.

preliminary exercise. \%MVIC measurement was repeated three times, holding each action for 10 seconds with the notice "Start" and one minute of rest was taken between each action. To minimize muscle fatigue, five minute rest was provided after intervention. During the exercise, $\%$ MVIC measurement were collected for 10 seconds and used for data analysis for 8 seconds in the middle except the first and last 1 seconds. Sequence of exercise was randomly selected.

\section{Statistical Analyses}

Paired T-test was used for comparison of the before-and-after neck flexion to analyze the muscle activity of the Biceps brachii of next flexion and extension, the SCM, and the Anterior deltoid during elbow flexion exercise. The Repeated measured ANOVA was used for comparing \%MVIC in each muscle. The significance level 
(?) was set at .05 and SPSS (version 25.0) was used for the statistical analysis of data collected with experiment.

\section{Results}

\section{General Characteristic of Subject}

The subjects participating in this study were total twenty adults; 15 men and 5 women, attending $\mathrm{K}$ university in Gyeongsangnam-do. The average age is $23.35 \pm 1.46$, the average height is $172.05 \pm 7.50 \mathrm{~cm}$, and the average weight is $67.70 \pm 12.79 \mathrm{~kg}$ (Table 1).

\section{Compared EMG Depend on Muscle Strength \% Section}

In the case of the Biceps brachii, EMG were $39.09 \pm$ 15.44 in maximal muscle strength $50 \sim 60 \%$ section, 36.43 \pm 13.12 in maximal muscle strength $60 \sim 70 \%$ section, and $35.30 \pm 15.21$ in maximal muscle strength $70-80 \%$ section

Table 1. General Characteristic of Subject $(\mathrm{N}=20)$

\begin{tabular}{cc}
\hline Variables & Subject $(\mathrm{N}=20)$ \\
\hline Sex (male / female) & $15 / 5$ \\
Age (years) & $23.35 \pm 1.46$ \\
Hight $(\mathrm{cm})$ & $172.05 \pm 7.50$ \\
Weight $(\mathrm{kg})$ & $67.70 \pm 12.79$ \\
\hline
\end{tabular}

Mean \pm Standard Deviation during neck flexion. When neck was in a neutral position, EMG were $36.19 \pm 12.13$ in maximal muscle strength $50 \sim 60 \%$ section, $35.50 \pm 13.83$ in maximal muscle strength $60 \sim 70 \%$ section, and $34.06 \pm 12.81$ in maximal muscle strength $70 \sim 80 \%$ section. EMG of the Biceps brachii did not have significant difference in accordance to maximal muscle strength $\%$ section $(p>.05)$ (Table 2).

In the case of the SCM, EMG were $37.51 \pm 14.71$ in maximal muscle strength 50 60\% section, $37.30 \pm 17.26$ in maximal muscle strength $60 \sim 70 \%$ section, and 34.18 \pm 15.32 in maximal muscle strength $70 \sim 80 \%$ section during neck flexion. When neck was in a neutral position, EMG were $39.01 \pm 11.72$ in $50 \sim 60 \%$ section, $34.54 \pm 13.84$ in maximal muscle strength $60 \sim 70 \%$ section, and 37.93 \pm 14.98 in maximal muscle strength $70-80 \%$ section. EMG of the SCM did not have significant difference in accordance to maximal muscle strength \% section ( $\mathrm{p}>$ .05) (Table 2).

In the case of the Anterior deltoid, EMG were 46.20 \pm 16.77 in $50 \sim 60 \%$ section, $43.57 \pm 18.77$ in maximal muscle strength $60 \sim 70 \%$ section, and $42.41 \pm 20.30$ in maximal muscle strength $70 \sim 80 \%$ section during neck flexion. When neck was in a neutral position, EMG were $41.08 \pm 14.53$ in maximal muscle strength $50-60 \%$ section, $41.60 \pm 21.04$ in $60 \sim 70 \%$ section, and $41.44 \pm 17.07$ in maximal muscle strength $70 \sim 80 \%$ section. EMG of the

Table 2. Compared EMG Depend Muscle Strength

\begin{tabular}{ccccccc}
\hline \multirow{2}{*}{ Muscle } & Position & \multicolumn{3}{c}{ Muscle strength } & F & $\mathrm{p}$ \\
\cline { 3 - 6 } & & $50-60 \%$ & $60-70 \%$ & $70-80 \%$ & & \\
\hline \multirow{2}{*}{ Biceps } & Flexion & $36.19 \pm 12.13$ & $35.50 \pm 13.83$ & $34.06 \pm 12.81$ & .39 & .67 \\
& Neutral & $39.09 \pm 15.44$ & $36.429 \pm 13.12$ & $35.30 \pm 15.21$ & 1.09 & .35 \\
\hline \multirow{2}{*}{ SCM } & Flexion & $37.51 \pm 14.71$ & $37.30 \pm 17.26$ & $34.18 \pm 15.32$ & 1.61 & .33 \\
& Neutral & $39.01 \pm 11.72$ & $34.54 \pm 13.84$ & $37.93 \pm 14.98$ & 1.16 & .22 \\
\hline \multirow{2}{*}{ Ant. deltoid } & Flexion & $46.20 \pm 16.77$ & $43.57 \pm 18.77$ & $42.41 \pm 20.30$ & .96 & .39 \\
& Neutral & $41.08 \pm 14.53$ & $41.60 \pm 21.04$ & $41.44 \pm 17.07$ & .01 & .98 \\
\hline
\end{tabular}

Mean \pm Standard Deviation

EMG: electromyography, SCM: sternocleidomastoid 
Table 3. Compared EMG Depend on Neck Position

\begin{tabular}{cccccc}
\hline Muscle & Maximal Muscle Strength & Neutral & Flexion & $\mathrm{t}$ & $\mathrm{p}$ \\
\hline \multirow{3}{*}{ Biceps } & $50-60 \%$ & $36.19 \pm 12.13$ & $39.09 \pm 15.44$ & -1.55 & .13 \\
& $60-70 \%$ & $35.50 \pm 13.83$ & $36.43 \pm 13.12$ & -.54 & .59 \\
& $70-80 \%$ & $34.06 \pm 12.81$ & $35.30 \pm 15.21$ & -.57 & .57 \\
\hline SCM & $50-60 \%$ & $39.01 \pm 11.72$ & $37.51 \pm 14.71$ & .57 & .57 \\
& $60-70 \%$ & $34.54 \pm 13.84$ & $37.30 \pm 17.26$ & -.71 & .48 \\
& $70-80 \%$ & $37.93 \pm 14.98$ & $34.18 \pm 15.32$ & 2.27 & .03 * \\
\hline \multirow{2}{*}{ Ant. } & $50-60 \%$ & $41.08 \pm 14.53$ & $46.20 \pm 16.77$ & -2.90 & $.00 *$ \\
Deltoid & $60-70 \%$ & $41.60 \pm 21.04$ & $43.57 \pm 18.77$ & -.92 & .36 \\
& $70-80 \%$ & $41.44 \pm 17.07$ & $42.41 \pm 20.30$ & -.63 & .53 \\
\hline
\end{tabular}

Mean \pm Standard Deviation, ${ }^{*} \mathrm{p}<.05$

EMG: electromyography, SCM: sternocleidomastoid

Anterior deltoid did not have significant difference in accordance to maximal muscle strength \% section $(\mathrm{p}>$ .05) (Table 2).

\section{Compared Biceps EMG Depend on Neck Position}

In the result of comparing the muscle activity according to neck flexion or not, EMG of the Biceps brachii were $36.19 \pm 12.13$ in maximal muscle strength $50-60 \%$ section, $35.50 \pm 13.83$ in maximal muscle strength $60 \sim 70 \%$ section, and $34.06 \pm 12.81$ in maximal muscle strength $70 \sim 80 \%$ section when neck was in a neutral position. During neck flexion, EMG were $39.09 \pm 15.44$ in maximal muscle strength $50 \sim 60 \%$ section, $36.43 \pm 13.12$ in maximal muscle strength $60 \sim 70 \%$ section, and $35.30 \pm 15.21$ in maximal muscle strength $70 \sim 80 \%$ section. EMG of the Biceps brachii did not have significant difference between neck in neutral position and neck flexion $(\mathrm{p}>.05)$ (Table 3$)$.

EMG of the SCM were $39.01 \pm 11.72$ in maximal muscle strength $50 \sim 60 \%$ section, $34.54 \pm 13.84$ in maximal muscle strength $60 \sim 70 \%$ section, and $37.93 \pm 14.98$ in maximal muscle strength $70 \sim 80 \%$ section when neck was in a neutral position. During neck flexion, EMG were $37.51 \pm 14.71$ in maximal muscle strength $50 \sim 60 \%$ section, $37.30 \pm 17.26$ in maximal muscle strength $60 \sim 70 \%$ section, and 34.18 \pm 15.32 in maximal muscle strength $70-80 \%$ section. EMG of SCM did not have significant difference between neck in neutral position and neck flexion $(\mathrm{p}>.05)$. However, EMG had a difference in \%MVIC 70-80 section between neck in neutral position and neck flexion $(\mathrm{p}<.05)$ (Table 3$)$.

EMG of the Anterior deltoid were $41.08 \pm 14.53$ in maximal muscle strength $50 \sim 60 \%$ section, $41.60 \pm 21.04$ in maximal muscle strength $60 \sim 70 \%$ section, and 41.44 \pm 17.07 in maximal muscle strength $70 \sim 80 \%$ section when neck was in a neutral position. During neck flexion, EMG were $46.20 \pm 16.77$ in maximal muscle strength $50 \sim 60 \%$ section, $43.57 \pm 18.77$ in maximal muscle strength $60 \sim 70 \%$ section, and $42.41 \pm 20.30$ in maximal muscle strength $70 \sim 80 \%$ section. EMG of the Anterior deltoid did not have significant difference between neck in neutral position and neck flexion $(\mathrm{p}>.05)$. However, EMG had a difference in \%MVIC 50-60 section between neck in neutral position and neck flexion $(\mathrm{p}<.05)$ (Table 3$)$.

\section{Discussion}

The purpose of this study was to determine the effect of applying neck flexion during elbow flexion exercise on 
muscle activity of the Biceps brachii, the Anterior deltoid and the SCM. However, the Anterior deltoid showed the highest EMG compared to other muscles in all sections. It means that the Anterior deltoid has the biggest impact on the elbow flexion. The Anterior deltoid is located in the middle of the Biceps brachii and SCM in anatomical position [8]. Due to this characteristic, it is thought that the activity of the Anterior deltoid was the highest during elbow bending as it served as a force transmitter. Furthermore, the Anterior deltoid is considered as an important muscle during the motion of upper limb and arm flexion. In the experiment with people who have shoulder problems, the preceding research also reported that muscular strength of the Anterior deltoid has a vital role in improving the motion of arm [12,13]. In the result of EMG in each muscle according to neck position, EMG increased in most of muscle during neck flexion rather than neck in neutral position except in maximal muscle strength $50 \sim 60 \%$ and $70 \sim 80 \%$ section of SCM EMG.

In the similar preceding research, the study regarding the effect of clenching on isometric exercise of upper limb shows that the muscular strength was significantly increased in the statistics when clenching is done rather than the opposite case [8]. In another research, the study regarding the effect of EMG change in Deltoid according to using mouth guard for the taekwondo athletes shows that Deltoid EMG was increased while using mouth guard rather than the opposite case and there is significant difference in the statistics [14].

During elbow flexion, the \%MVIC value in the neutral position of the neck was measured to be $37.931 \pm 14.981$, and the \%MVIC value of the neck flexion position decreased significantly to $34.180 \pm 15.320$. When the SCM is excessively strengthened during elbow flexion, the instability increases during neck movement due to weakening of the deep neck flexors, which can cause neck pain [15]. When the muscle length is shortened, the muscle activity may increase. In the upper trapezius, turning the neck in the ipsilateral direction while turning a shoulder externally decreased the muscle activity significantly, but the muscle activity was increased significantly by turning the neck in the contralateral direction. On the other hand, in the lower trapezius, turning the neck in the ipsilateral direction increased the muscle activity significantly, but the muscle activity was decreased significantly by turning the neck in the contralateral direction decreased it significantly [16]. It is thought that the neck flexion position increased the activity of the deep neck flexion muscles, and thus the muscle activity of the SCM was reduced. In particular, when a strong strength is mobilized, the compensating of SCM can occur strongly, and it is thought that the neck bending posture prevented it.

Although the measuring level is different, the action on head-neck level seemed to affect the EMG of upper limb and showed the similarity to the result of this study. In the preceding research, many of muscles are in the Spine, Scapula, Clavicle, and Humerus and affect by connecting different joints directly and indirectly [1]. The preceding research for the correlation between body muscle and shoulder muscle showed the effect on shoulder elevator muscle of the flexion and extension of trunk [17]. As a result, the flexion posture of spine weakens elevator muscle on shoulder compared to a standing posture. In the result of EMG of middle Deltoid in flexion and standing posture, the muscle activity of middle Deltoid was significantly higher in the flexion posture rather than standing posture. This showed the result consistent with the study of researcher that the flexion and extension exercise of trunk have an effect on the shoulder muscles. Also, in the research applied the Maitland Manual technique the patient who has a chronic pain reported that the patient's pain was reduced and isometric muscular strength of the Biceps brachii was increased $[18,19]$. The result of study above showed the manual technique for neck affected the upper limbs muscular strength and also similar to the result of this study that the upper limbs muscular strength was 
affected according to the neck position. In accordance with the preceding research, this was consistent with the result that human body is directly and indirectly connected and influences each other [20]. The limitation of this study is that it is difficult to generalize the result of study because of the small number of subjects. In addition, there were many of studies on the activity of upper limb muscle during shoulder spread but studies on upper limb muscle during elbow flexion were not enough. In the following studies, those imitations above should be supplemented.

Neck flexion action applied during powerful arm flexion exercise shows to increase the muscle activity of arm and to decrease the action of neck muscle. Therefore, neck flexion during arm flexion exercise needed strong strength seems effective in increasing arm muscular strength and suppressing of compensation of neck.

\section{Conclusion}

Neck flexion action applied during powerful arm flexion exercise shows to increase the muscle activity of arm and to decrease the action of neck muscle. Therefore, neck flexion during arm flexion exercise needed strong strength seems effective in increasing arm muscular strength and suppressing of compensation of neck.

\section{References}

[1] Kim DH, Choi JD, Oh JS. Analysis of Muscle Activity of the Scapular Rotators With Changes of Shoulder Abduction Angle in Both Erect and Slouched Trunk Position. Physical Therapy Korea. 2004;11(1):45-52.

[2] Lee HE, Yang JK, Son JH, et al. Difference of Pinch Strength According to Testing Posture and Various Flexion Degree of Elbow Joint. Journal of International Academy of Physical Therapy Research. 2010;1(2):113-9.

[3] Majsak MJ. Application of motor learning principles to the stroke population. Topics in Stroke Rehabilitation.
1996;3(2):37-59.

[4] Grgic J, Lazinica B, Garofolini A, et al. The effects of time of day-specific resistance training on adaptations in skeletal muscle hypertrophy and muscle strength: a systematic review and meta-analysis. Chronobiology international. 2019;36(4):449-60.

[5] McCartney N, Hicks AL, Martin J, et al. A longitudinal trial of weight training in the elderly: continued improvements in year 2. The Journals of Gerontology Series A: Biological Sciences and Medical Sciences. 1996;51(6):B425-33.

[6] Holmes JW. Teaching from classic papers: Hill's model of muscle contraction. Advances in physiology education. 2006;30(2):67-72.

[7] Sprague K, Reynolds B. The Gold's gym book of bodybuilding. McGraw-Hill/Contemporary. 1983.

[8] Myers TW. Anatomy trains: myofascial meridians for manual and movement therapists. Korea. Elsevier Health Sciences. 2013.

[9] Cho DW, Seo KW, Yoon YJ, et al. Simulation of one arm curl action. Journal of Physical Education \& Sports Science. 1997;13(1997):199-210.

[10] Sahrmann SA. Diagnosis and treatment of movement impairment syndromes. Korea. Elsevier Health Sciences. 2002.

[11] Choung Sd, Weon Jh, Jung Dy. Effect of movement plane and shoulder flexion angle on scapular upward rotator during scapular protraction exercise. Journal of Korean Society of Physical Medicine. 2013;8(1):41-8.

[12] Hintermeister RA, Lange GW, Schultheis JM, et al. Electromyographic activity and applied load during shoulder rehabilitation exercises using elastic resistance. The American Journal of Sports Medicine. 1998;26(2): 210-20.

[13] Levy O, Mullett H, Roberts S, et al. The role of anterior deltoid reeducation in patients with massive irreparable degenerative rotator cuff tears. Journal of shoulder and elbow surgery. 2008;17(6):863-70. 
[14] Lee SJ. A study on the change of deltoid muscle strength wearing mouth guard. The Korean Journal of Oral and Maxillofacial Pathology. 2010;34(4):201-6.

[15] Lee BK, Seo DK. The importance of optimal gaze direction on deep neck flexor activation in chronic neck pain. Heathcare. 2020;8(4):449.

[16] Shin YW, Kim CH, Han JT. Effect of neck rotating directions on the muscle activity of upper and lower trapezius during shoulder external rotation. The Journal of Korean Physical Therapy. 2020;32(2):101-6.

[17] Massie CL, Fritz S, Malcolm MP. Elbow extension predicts motor impairment and performance after stroke.
Rehabilitation research and practice. 2011;2011(1):1-7.

[18] Maitland G, Hengeveld E, Banks K, et al. Maitland's vertebral manipulation. Edinburgh. Churchill Livingston. 2005.

[19] Kebaetse M, McClure P, Pratt NA. Thoracic position effect on shoulder range of motion, strength, and three-dimensional scapular kinematics. Archives of physical medicine and rehabilitation. 1999;80(8):945-50.

[20] Suter E, McMorland G. Decrease in elbow flexor inhibition after cervical spine manipulation in patients with chronic neck pain. Clinical Biomechanics. 2002;17(7):541-4. 